Wolling, Jens:

Entwicklungstrends in der Computerspielnutzung bei Kindern, Jugendlichen und Erwachsenen

Original erschienen in:

Quandt, Thorsten; Wimmer, Jeffrey; Wolling, Jens (Hrsg.): Die Computerspieler : Studien zur Nutzung von Computergames. - Wiesbaden: VS, Verl. für Sozialwiss., 2008, S. 73-93.

ISBN (print): 3-531-15085-5

ISBN (online): 978-3-531-90823-5

DOI: $\quad$ 10.1007/978-3-531-90823-6_5

URL: $\quad$ http://dx.doi.org/10.1007/978-3-531-90823-6_5

[Gesehen: 08.12.2015] 


\title{
Entwicklungstrends in der Computerspielnutzung bei Kindern, Jugendlichen und Erwachsenen
}

\author{
Jens Wolling
}

\section{$1 \quad$ Einleitung}

Wie groß ist der Anteil der Kinder, Jugendlichen und Erwachsenen, die ihre Freizeit mit Computerspielen verbringen? Haben Computerspiele in allen Bevölkerungsgruppen an Popularität gewonnen? Sind es vor allem die Hauptschüler, die ihre Zeit mit dem Spielen am Computer verbringen? Wie hat sich die Nutzung von Online- und Handyspielen in den zurückliegenden Jahren entwickelt? Um solche Fragen zu beantworten, benötigt man Daten aus repräsentativen Trendstudien. Die meisten Untersuchungen zur Nutzung von Computerspielen beruhen jedoch auf einmaligen Querschnitterhebungen und verwenden Stichproben aus kleinen Grundgesamtheiten oder Daten aus nicht-repräsentativen Gelegenheitsstichproben. Aufgrund forschungsökonomischer Restriktionen wird sich dies auch in $\mathrm{Zu}-$ kunft nicht grundsätzlich ändern. In vielen Fällen ist die fehlende oder eingeschränkte Repräsentativität kein gravierendes Problem, in anderen wäre es aber durchaus wünschenswert, Aussagen darüber treffen zu können, in wie weit die ermittelten Ergebnisse verallgemeinerbar sind.

Um die Reichweite von Befunden aus nicht-repräsentativen Erhebungen besser einschätzen zu können, besteht die Möglichkeit, die grundlegenden Strukturmerkmale der jeweils realisierten Stichprobe mit den Befunden aus repräsentativen Erhebungen zu vergleichen. Die dabei beobachtbaren Abweichungen geben dann Hinweise darauf, mit welchen Verzerrungen zu rechnen ist. Allerdings sind die dafür notwendigen repräsentativen Vergleichsdaten nicht ohne weiteres zugänglich. Zwar findet man in vielen Beiträgen einzelne Ergebnisse zur Computerspielnutzung aus unterschiedlichen (bevölkerungs)repräsentativen Erhebungen (z.B. Opaschowski 1999, 45 ff.; Schlütz 2002, 18 f.; Klimmt 2004: 696; Emmer 2005, 117 ff.; van Eimeren \& Frees 2005, 371; Langness, Leven \& Hurrelmann 2006, 78 ff.), eine systematische Überblicksdarstellung repräsentativer Nutzungszahlen liegt aber bislang nicht vor.

Dank der Unterstützung durch das Institut für Demoskopie Allensbach und durch den Medienpädagogischen Forschungsverbund Südwest (mpfs) ${ }^{1}$ ist es an dieser Stelle möglich, Daten zu präsentieren, die einen breit angelegten Überblick über die Nutzung von Computerspielen in Deutschland vermitteln. Dabei handelt es sich um Ergebnisse aus der ACTA

Ich danke Dr. Peter Voß und Thomas Rathgeb für die Unterstützung bei der Anfertigung dieses Beitrags. Dr. Voß hat die relevanten ACTA-Trenddaten zusammengestellt und von Thomas Rathgeb wurden die Auswertungen der JIM- und KIM-Studien vorgenommen. 
(Allensbacher Computer- und Technik-Analyse) sowie um Befunde aus den Studien KIM (Kinder und Medien) und JIM (Jugend, Information, (Multi-)Media). Die drei Untersuchungen ergänzen sich sehr gut, da mit den Daten von KIM und JIM die Computerspielnutzung von Kindern und Jugendlichen abgebildet wird, während die ACTA Befunde zum Umgang der Erwachsenen mit Computerspielen bereitstellt. Besonders wertvoll sind die Daten durch die Tatsache, dass es sich bei allen drei Studien um Längsschnittuntersuchungen handelt, in deren Rahmen groß angelegte Befragungen in regelmäßigen Abständen durchgeführt werden. ${ }^{2}$ Dadurch ist es möglich, Nutzungstrends zu identifizieren. Die nachfolgend vorgestellten Ergebnisse sind bislang nur in Teilen und verstreut veröffentlicht worden (z.B. Feierabend \& Klingler 1999, 2000, 2001, 2002, 2003a und 2003b; Feierabend \& Rathgeb 2005).

Begonnen wird mit der Darstellung der Ergebnisse zur Computerspielnutzung der Jugendlichen (JIM-Studie; s. Abschnitt 2). Die Jugendlichen werden als Referenzpunkt gewählt, weil die Beschäftigung mit Computerspielen in dieser Altersgruppe besonders verbreitet ist. Darauf hin werden die Befunde aus der Kinderstudie (KIM; s. Abschnitt 3) und abschließend dann die Nutzungsdaten der Erwachsenen (ACTA; s. Abschnitt 4) präsentiert. ${ }^{3}$ Die jeweiligen Ergebnisse werden in zeitlicher, sachlicher und sozialer Hinsicht differenziert.

\section{Zeitliche Differenzierung}

Alle drei Studien sind langfristig angelegt. Dadurch kann gezeigt werden werden, wie sich die Nutzung von Computerspielen in den zurückliegenden Jahren entwickelt hat.

\section{Sachliche Differenzierung}

Der Gegenstand der Betrachtung - die unterschiedlichen Varianten der Computerspielenutzung - lässt sich in vielfacher Weise ausdifferenzieren. Im Rahmen dieses Beitrags wird zwischen dem Spielen am Computer allgemein, Onlinespielen und Spielen auf dem Handy unterschieden. Weitergehende Differenzierungen in sachlicher Hinsicht - beispielsweise hinsichtlich der Nutzung einzelner Genres oder Plattformen (Konsole / PC) - sind aufgrund der Datenlage nur vereinzelt möglich.

\section{Soziale Differenzierung}

Die soziale Differenzierung dient dazu, die Computerspieler genauer zu beschreiben. Wie unterscheiden sich die verschiedenen soziodemographischen Gruppen hinsichtlich ihres Spielverhaltens? In der Zusammenschau der verschiedenen Datenquellen entsteht so ein Gesamtbild der Entwicklung der Spielnutzung in allen Altersgruppen der Bevölkerung.

2 Bei der ACTA handelt es sich um eine mündliche face to face Befragung mit standardisiertem Fragebogen Die Stichprobe wird nach dem Quoten-Auswahlverfahren gebildet und ist repräsentativ für die deutsche Bevölkerung zwischen 14 und 64 Jahren in Privathaushalten. Bei der JIM-Studie handelt es sich um eine computergestützte telefonische Befragung (CATI). Die Studie ist repräsentativ für Jugendliche zwischen 12 und 19 Jahren in Telefonhaushalten der BRD. Bei der KIM-Studie handelt es sich um eine repräsentative mündliche face to face Befragung von deutschsprachigen Kindern zwischen sechs und 13 Jahren in der BRD sowie einer schriftlich Befragung von deren primärer Erziehungsperson (meistens die Mutter).

3 Prinzipiell wäre es wünschenswert gewesen, die Ergebnisse der Studie stärker zu integrieren. Aufgrund der unterschiedlichen Befragungsinstrumente und Auswertungsstrategien der Institute war dies aber nicht möglich 
Ein wichtiges Anliegen des Beitrags ist die Bereitstellung möglichst umfangreichen Datenmaterials. Um dies zu gewährleisten und gleichzeitig den Rahmen des Aufsatzes nicht zu sprengen, werden jeweils nur die auffälligsten Befunde aus den Tabellen herausgegriffen und erörtert.

\section{Entwicklung der Computerspielnutzung bei Jugendlichen}

Nur diejenigen Jugendlichen, die überhaupt einen Computer verwenden, sind auch potenzielle Computerspieler. Deswegen muss die Entwicklung der Spielnutzung vor dem Hintergrund der Daten zur Computernutzung interpretiert werden. Dabei zeigt sich, dass sowohl bei Jungen und Mädchen als auch bei Schülern aller Schultypen die Nutzung des Computers kontinuierlich zugenommen hat (Tabelle 1). Dasselbe gilt für die verschiedenen Altersgruppen. Mittlerweile ist der Anteil der Jugendlichen ohne Computererfahrung in fast allen Segmenten deutlich unter $10 \%$ gesunken. Auffällig ist zudem, dass sich im Untersuchungszeitraum die vorhandenen Nutzungsklüfte wesentlich abgeschwächt haben - und zwar sowohl zwischen den Geschlechtern als auch hinsichtlich der Bildung.

Tabelle 1: Computernutzung durch Jugendliche (JIM-Studie): Prozent aller Jugendlichen, die den Computer ,mindestens einmal im Monat' nutzen (Computernutzer)

\begin{tabular}{rrrrrrrrr}
\hline Jahr & $\mathbf{1 9 9 8}$ & $\mathbf{1 9 9 9}$ & $\mathbf{2 0 0 0}$ & $\mathbf{2 0 0 1}$ & $\mathbf{2 0 0 2}$ & $\mathbf{2 0 0 3}$ & $\mathbf{2 0 0 4}$ & $\mathbf{2 0 0 5}$ \\
$\mathrm{n}=$ & 803 & 1204 & 1200 & $2018^{4}$ & 1092 & 1209 & 1000 & 1203 \\
\hline Gesamt & 71 & 76 & 81 & 83 & 93 & 93 & 94 & 95 \\
\hline Mädchen & 63 & 70 & 76 & 79 & 92 & 90 & 92 & 94 \\
Jungen & 78 & 82 & 86 & 87 & 94 & 95 & 95 & 95 \\
\hline 12-13 J. & 71 & 77 & 78 & 83 & 90 & 91 & 93 & 93 \\
14-15 J. & 74 & 80 & 85 & 87 & 95 & 94 & 93 & 97 \\
16-17 J. & 72 & 74 & 83 & 83 & 92 & 93 & 93 & 97 \\
$18-19$ J. & 67 & 73 & 77 & 79 & 94 & 93 & 96 & 92 \\
\hline Hauptschule & 58 & 65 & 69 & 72 & 84 & 88 & 89 & 89 \\
Realschule & 72 & 77 & 83 & 81 & 93 & 92 & 94 & 95 \\
Gymnasium & 78 & 82 & 85 & 91 & 97 & 95 & 96 & 98 \\
\hline
\end{tabular}

Betrachtet man nun die Entwicklung der Computerspielnutzung, dann fällt auf, dass der Anteil der Spieler von $53 \%$ auf $38 \%$ deutlich zurückgegangen ist (Tabelle 2). Bei der Interpretation dieses Befundes ist aber zu bedenken, dass dabei nicht die Jugendlichen insgesamt, sondern nur die Computernutzer die Basis der Prozentuierung bilden. Wenn man dies berücksichtigt, dann stellt man fest, dass der Anteil der Computerspieler auf alle Ju-

Die Stichprobe 2001 umfasst auch eine Zusatzstichprobe von Jugendlichen aus Baden Württemberg und Rheinland-Pfalz (Aufstockung auf je 500), die aber für die Auswertung heruntergewichtet wurde (Feierabend \& Klingler 2002) 
gendlichen bezogen weitgehend konstant geblieben ist. 1998 waren $37 \%$ der Jugendlichen Spieler, 2005 waren es $36 \%$.

In den verschiedenen soziodemographischen Segmenten waren die Entwicklungen aber nicht einheitlich: Während bei den Jungen der relative Anteil der Spieler an den Computernutzern weitgehend konstant blieb und damit die absolute Zahl der männlichen Spieler zunahm, ist bei den Mädchen sowohl die relative als auch die absolute Spielerzahl kleiner geworden. In den verschiedenen Altersgruppen sind Reichweitenverluste vor allem bei den Jüngeren zu verzeichnen, bei den älteren Spielern ist die absolute Zahl der Nutzer hingegen gestiegen. Im Bezug auf die Schulbildung der Spieler ist festzuhalten, dass der relative Nutzeranteil bei allen drei Schultypen geringer geworden ist, der absolute Anteil hat sich hingegen kaum verändert.

Wenn man nun nicht so sehr auf die Entwicklung der Nutzerzahlen achtet, sondern mehr auf die Unterschiede zwischen den Nutzergruppen, dann stellt man fest, dass (a) deutlich mehr Jungen als Mädchen häufig mit dem Computer spielen, dass (b) unter den Jüngeren mehr Vielspieler sind als unter den Älteren und das (c) Hauptschüler häufiger als Realschüler und diese wiederum häufiger als Gymnasiasten ihre Zeit mit Computerspielen verbringen. In nicht repräsentativen Studien haben viele der Befragten oft einen relativ hohen Bildungsabschluss, sodass teilweise verallgemeinernd vom „hochgebildeten Gamer“ (Theunert, Demmler \& Kirchhoff 2002, 142) die Rede ist. Angesichts der hier präsentierten Zahlen müssen solche Aussagen sicherlich relativiert werden. Bemerkenswert ist aber auch, dass die schulform- und altersbedingten Unterschiede im Zeitverlauf tendenziell eher geringer geworden sind.

Tabelle 2: Computerspielnutzung durch Jugendliche (JIM-Studie): Prozent der Computernutzer, die ,mehrmals pro Woche' oder ,täglich' am Computer spielen

\begin{tabular}{rrrrrrrrr}
\hline Jahr & $\mathbf{1 9 9 8}$ & $\mathbf{1 9 9 9}$ & $\mathbf{2 0 0 0}$ & $\mathbf{2 0 0 1}$ & $\mathbf{2 0 0 2}$ & $\mathbf{2 0 0 3}$ & $\mathbf{2 0 0 4}$ & $\mathbf{2 0 0 5}$ \\
$\mathrm{n}=$ & 568 & 913 & 969 & 1676 & 1013 & 1121 & 935 & 1142 \\
\hline Gesamt & 53 & 56 & 48 & 49 & 44 & 45 & 41 & 38 \\
\hline Mädchen & 37 & 39 & 28 & 30 & 21 & 24 & 20 & 15 \\
Jungen & 66 & 70 & 65 & 65 & 66 & 64 & 61 & 61 \\
\hline 12-13 J. & 66 & 71 & 59 & 68 & 60 & 58 & 47 & 43 \\
14-15 J. & 71 & 63 & 59 & 55 & 50 & 53 & 48 & 48 \\
$16-17$ J. & 48 & 52 & 41 & 40 & 39 & 38 & 41 & 34 \\
$18-19$ J. & 26 & 39 & 31 & 30 & 27 & 27 & 31 & 30 \\
\hline Hauptschule & 63 & 65 & 61 & 52 & 46 & 53 & 53 & 43 \\
Realschule & 56 & 57 & 46 & 50 & 46 & 47 & 39 & 39 \\
Gymnasium & 47 & 51 & 44 & 47 & 42 & 38 & 38 & 36 \\
\hline
\end{tabular}

Spielen ist nicht immer - aber doch sehr häufig - eine gemeinschaftliche Tätigkeit, und auch beim Computerspielen gibt es vielfach die Option (bei manchen sogar die Notwendigkeit), gemeinsam mit anderen zu spielen. Selbst bei jenen Spielen, die über keinen Mul- 
tiplayermodus verfügen, besteht die Möglichkeit, sich mit Freunden zum Computerspielen zu treffen und sich dann beim Spielen am Computer abzuwechseln. LAN-Partys und verschiedene Formen von Onlinespielen sind weitere Formen des gemeinsamen Spiels. In der JIM-Studie wurden die verschiedenen Varianten des gemeinsamen Computerspielens nicht ermittelt. Von daher ist nicht zu entscheiden, auf welche Formen des gemeinsamen Spielens sich die Antworten jeweils beziehen. Für die Interpretation der Befunde wäre dies aber durchaus interessant, denn es zeigt sich relativ deutlich, dass die Bedeutung des gemeinsamen Spiels rückläufig ist (Tabelle 3). Und zwar in doppelter Hinsicht: Zum einen sinkt der Anteil der Jugendlichen, die überwiegend gemeinsam spielen, und zum anderen wird auch der Prozentanteil derjenigen geringer, die sagen, dass sie beides gleich häufig machen. Gerade angesichts der Tatsache, dass sich das Angebot an Multiplayer-Spielen in den zurückliegenden Jahren ohne Zweifel erheblich vergrößert hat, ist dies sicherlich ein bemerkenswerter Befund. Der Rückgang könnte auf die zunehmende Diffusion der Computertechnologie zurückzuführen sein: Für viele Jugendliche besteht einfach nicht mehr die Notwendigkeit, bei/mit anderen zu spielen, da sie mittlerweile selbst einen Computer haben und nicht mehr zu Freunden gehen müssen, wenn sie spielen wollen. Die nachlassende Bedeutung des gemeinsamen Spiels und die steigende Anzahl der Einzelspieler sind sowohl bei den Mädchen als auch bei den Jungen festzustellen, allerdings sind die Tendenzen bei den Jungen etwas ausgeprägter. Im Ergebnis führte dies dazu, dass im Jahr 2005 in dieser Hinsicht kaum noch Unterschiede zwischen den Geschlechtern festzustellen sind.

Tabelle 3: Computerspiel allein oder zusammen (JIM-Studie): Prozent aller Computernutzer, die überwiegend allein oder überwiegend mit anderen spielen

\begin{tabular}{lrrrrrrrrr}
\hline & Jahr & $\mathbf{1 9 9 8}$ & $\mathbf{1 9 9 9}$ & $\mathbf{2 0 0 0}$ & $\mathbf{2 0 0 1}$ & $\mathbf{2 0 0 2}$ & $\mathbf{2 0 0 3}$ & $\mathbf{2 0 0 4}$ & $\mathbf{2 0 0 5}$ \\
& $\mathrm{n}=$ & 517 & 823 & 842 & 1383 & 840 & 922 & 721 & 846 \\
\hline Computerspielen & Gesamt & 26 & 28 & 30 & 35 & 35 & 38 & 48 & 46 \\
überwiegend allein & Mädchen & 33 & 31 & 34 & 39 & 38 & 36 & 49 & 49 \\
& Jungen & 21 & 27 & 28 & 33 & 33 & 40 & 48 & 45 \\
\hline Computerspielen & Gesamt & 20 & 17 & 17 & 18 & 15 & 14 & 12 & 15 \\
überwiegend mit & Mädchen & 23 & 16 & 17 & 19 & 14 & 17 & 12 & 14 \\
anderen & Jungen & 18 & 18 & 17 & 17 & 15 & 11 & 12 & 15 \\
\hline
\end{tabular}

Die Frage zu den Lieblingsgenres im Bereich der Computerspiele wurde den Jugendlichen zum ersten Mal im Jahr 2000 gestellt: Die Daten belegen, dass die sechs berücksichtigten Spielgenres sich hinsichtlich ihrer Attraktivität deutlich unterscheiden. Diese Unterschiede in den Genrepräferenzen veränderten sich während der Untersuchungszeit nicht grundsätzlich, sondern nur graduell (Tabelle 4). Die größte Popularität haben die Strategiespiele, die geringste die Jump'n'Runs. Zum Teil findet man, dass die Vorlieben der Geschlechter erheblich variieren: Action- und Simulationsspiele werden von den Jungen in allen Untersuchungsjahren wesentlich stärker bevorzugt. Bei den Actionspielen sind die Vorlieben im Laufe der Zeit sogar noch weiter auseinandergedriftet: mehr Jungen bezeichnen Actionspiele als ihre Favoriten, bei den Mädchen sinkt der Anteil tendenziell. 
Tabelle 4: Computerspielgenrepräferenzen der Jugendlichen (JIM-Studie): Prozent der Computernutzer, die das jeweilige Genre genannt haben

\begin{tabular}{rrrrrrrr}
\hline Lieblingsspielgenre & Jahr & $\mathbf{2 0 0 0}$ & $\mathbf{2 0 0 1}$ & $\mathbf{2 0 0 2}$ & $\mathbf{2 0 0 3}$ & $\mathbf{2 0 0 4}$ & $\mathbf{2 0 0 5}$ \\
bis zu 3 Nennungen & $\mathrm{n}=$ & 842 & 1383 & 840 & 922 & 721 & 846 \\
\hline Strategie & Mädchen & 49 & 60 & 67 & 70 & 71 & 70 \\
& Jungen & 55 & 58 & 47 & 55 & 50 & 53 \\
\hline \multirow{2}{*}{ Action } & Mädchen & 24 & 23 & 24 & 23 & 17 & 19 \\
& Jungen & 42 & 43 & 56 & 58 & 58 & 55 \\
\hline Simulation & Mädchen & 16 & 17 & 23 & 19 & 24 & 21 \\
& Jungen & 48 & 40 & 42 & 37 & 49 & 41 \\
\hline \multirow{2}{*}{ Adventure } & Mädchen & 22 & 15 & 17 & 21 & 15 & 10 \\
& Jungen & 22 & 12 & 15 & 8 & 7 & 8 \\
\hline Rollenspiel & Mädchen & 2 & 0 & 5 & 5 & 12 & 9 \\
& Jungen & 10 & 10 & 12 & 9 & 10 & 11 \\
\hline Jump'n'Run & Mädchen & 3 & 5 & 3 & 4 & 4 & 1 \\
& Jungen & 1 & 2 & 0 & 1 & 2 & 1 \\
\hline
\end{tabular}

Bei den Simulationen ist hingegen eher ein Annäherungsprozess zu beobachten. Die Strategie- und Rollenspiele wiederum haben in den sechs betrachteten Jahren bei den Mädchen an Attraktivität gewonnen, während sich bei den Jungen keine Veränderungen zeigen. Teilweise schwanken die Präferenzen zwischen den Jahren recht deutlich. Das kann auf verschiedene Faktoren zurückzuführen sein: Zum einen kann es sich um Stichprobenfehler handeln, zum anderen kann dafür aber auch der Markteintritt attraktiver Titel verantwortlich sein. ${ }^{5}$ Und schließlich ist zu bedenken, dass es sich bei diesen Kategorien um sehr breite und keinesfalls immer trennscharfe Genrebezeichnungen handelt, die den Befragten einigen Interpretationsspielraum lassen.

Wie oben bereits angemerkt, ist die Möglichkeit zur Computernutzung die Voraussetzung dafür, dass man das Gerät als Spielmedium verwendet. Um mit dem Computer Onlinespiele spielen zu können, muss zusätzlich die Gelegenheit zur Onlinenutzung gegeben sein. Deswegen muss - bevor die Befunde zur Onlinespielnutzung interpretiert werden können - zunächst betrachtet werden, wie sich der Anteil der Onliner in den verschiedenen Bevölkerungsgruppen entwickelt hat (Tabelle 5). Der Befund ist eindeutig: In allen betrachteten Gruppen ist der Anteil der Onliner sehr deutlich gestiegen. Die Unterschiede zwischen den Schülern der verschiedenen Schultypen sind in allen Jahren nachzuweisen; die zwischen Mädchen und Jungen sind hingegen im Laufe der Jahre verschwunden. Betrachtet man die Altersgruppen, dann findet man nur wenig Varianz. Allein bei den 12- bis 13Jährigen ist der Anteil der Onliner immer um gut zehn Prozentpunkte geringer als bei allen anderen.

Die Ergebnisse von Quandt \& Wimmer in diesem Band sprechen für diese Interpretation. 
Tabelle 5: Onlinenutzung durch Jugendliche (JIM-Studie): Prozent aller Jugendlichen, die zumindest, selten' Online gehen (Onliner)

\begin{tabular}{rrrrrrrrr}
\hline Jahr & $\mathbf{1 9 9 8}$ & $\mathbf{1 9 9 9}$ & $\mathbf{2 0 0 0}$ & $\mathbf{2 0 0 1}$ & $\mathbf{2 0 0 2}$ & $\mathbf{2 0 0 3}$ & $\mathbf{2 0 0 4}$ & $\mathbf{2 0 0 5}$ \\
$\mathrm{n}=$ & 803 & 1204 & 1200 & 2018 & 1092 & 1209 & 1000 & 1203 \\
\hline Gesamt & 18 & 29 & 57 & 63 & 83 & 81 & 84 & 86 \\
\hline Mädchen & 14 & 23 & 51 & 59 & 83 & 81 & 84 & 86 \\
Jungen & 21 & 35 & 62 & 67 & 83 & 87 & 86 & 87 \\
\hline 12-13 J. & 12 & 20 & 47 & 53 & 74 & 77 & 75 & 77 \\
14-15 J. & 19 & 32 & 59 & 70 & 85 & 86 & 85 & 88 \\
16-17 J. & 20 & 30 & 62 & 66 & 85 & 86 & 88 & 92 \\
18-19 J. & 20 & 33 & 60 & 63 & 89 & 88 & 91 & 87 \\
\hline Hauptschule & 11 & 20 & 45 & 49 & 69 & 74 & 75 & 71 \\
Realschule & 16 & 24 & 53 & 58 & 80 & 83 & 84 & 87 \\
Gymnasium & 23 & 38 & 66 & 74 & 92 & 90 & 91 & 94 \\
\hline
\end{tabular}

Vor diesem Hintergrund sind die in Tabelle 6 präsentierten Zahlen zu lesen: Sie zeigen auf den ersten Blick, dass sich der Anteil der Onlinespieler an den Onlinern im betrachteten Zeitraum nicht gravierend verändert hat. Zwischen neun und $15 \%$ der jugendlichen Onliner geben an, ,täglich' oder ,mehrmals die Woche' online zu spielen. Hinter diesem relativ stabilen Anteil verbirgt sich aber ein enormes absolutes Wachstum, denn die Zahl der Onliner ist in dem betrachteten Zeitraum um fast $500 \%$ gestiegen. Dass Onlinespiele in kurzer Zeit so schnell an Zuspruch gewinnen konnten, ist sicherlich vor allem auf die schnelleren (DSL) und preisgünstigeren (Flatrate) Internetzugänge zurückzuführen. Wie schon bei den Computerspielern generell, findet man bei den Onlinespielern Nutzungsunterschiede zwischen den Geschlechtern, die hier sogar noch deutlicher ausfallen.

Computerspiele können nicht nur am PC, sondern auch auf anderen Plattformen gespielt werden. Von besonderer Bedeutung - insbesondere für Jugendliche - sind dabei die Spielkonsolen. Der Anteil der Jugendlichen, die eine Spielkonsole besitzen, ist von 1998 bis 2005 um über $50 \%$ gestiegen (Tabelle 7).

Die zahlreichen Hardware-Neuerscheinungen, die insbesondere seit der Jahrtausendwende auf dem Markt platziert wurden (PS2, XBox, Game Cube, XBox 360, Playstation 3 etc.), haben zu dieser Entwicklung sicherlich entscheidend beigetragen. Das relativ kontinuierliche Wachstum ist in allen soziodemographischen Gruppen zu beobachten. Die schon bei den PC- und Onlinespielen festgestellten Unterschiede zwischen Mädchen und Jungen finden sich auch bei den Konsolen. Beim Alter sind die Zusammenhänge etwas anders. In den drei jüngeren Altersgruppen ist der Anteil der Konsolenbesitzer sehr ähnlich, nur bei den über 18-Jährigen ist er im gesamten Zeitverlauf geringer. Schaut man auf den Schultyp, dann findet man das gleiche Muster wie bei den PC-Spielen generell: Unter den Hauptschülern sind mehr Konsolenbesitzer als unter den Realschülern, und bei denen wiederum gibt es mehr Konsolenspieler als unter den Gymnasiasten. 
80

Tabelle 6: Nutzung von Onlinespielen durch Jugendliche (JIM-Studie): Prozent der Onliner, die ,mehrmals pro Woche' oder ,täglich' Onlinespiele nutzen

\begin{tabular}{rrrrrrrrr}
\hline Jahr & $\mathbf{1 9 9 8}$ & $\mathbf{1 9 9 9}$ & $\mathbf{2 0 0 0}$ & $\mathbf{2 0 0 1}$ & $\mathbf{2 0 0 2}$ & $\mathbf{2 0 0 3}$ & $\mathbf{2 0 0 4}$ & $\mathbf{2 0 0 5}$ \\
$\mathrm{n}=$ & 142 & 347 & 681 & 1272 & 904 & 1017 & 850 & 1040 \\
\hline Gesamt & 15 & 14 & 9 & 14 & 11 & 11 & 9 & 13 \\
\hline Mädchen & 5 & 9 & 5 & 6 & 2 & 3 & 3 & 3 \\
Jungen & 22 & 18 & 12 & 21 & 20 & 19 & 14 & 22 \\
\hline $12-13 \mathrm{~J}$. & 26 & 14 & 12 & 16 & 13 & 10 & 3 & 11 \\
$14-15 \mathrm{~J}$. & 16 & 21 & 9 & 18 & 11 & 13 & 5 & 12 \\
$16-17 \mathrm{~J}$. & 20 & 8 & 6 & 12 & 11 & 12 & 12 & 13 \\
$18-19 \mathrm{~J}$. & 5 & 14 & 10 & 9 & 9 & 10 & 7 & 15 \\
\hline Hauptschule & 13 & 28 & 12 & 17 & 16 & 17 & 10 & 16 \\
Realschule & 16 & 18 & 11 & 15 & 11 & 12 & 10 & 11 \\
Gymnasium & 15 & 8 & 7 & 13 & 10 & 10 & 7 & 13 \\
\hline
\end{tabular}

Tabelle 7: Besitz einer Spielkonsole bei Jugendlichen (JIM-Studie): Prozent aller Jugendlichen, die eine Spielkonsole besitzen

\begin{tabular}{rrrrrrrrr}
\hline Jahr & $\mathbf{1 9 9 8}$ & $\mathbf{1 9 9 9}$ & $\mathbf{2 0 0 0}$ & $\mathbf{2 0 0 1}$ & $\mathbf{2 0 0 2}$ & $\mathbf{2 0 0 3}$ & $\mathbf{2 0 0 4}$ & $\mathbf{2 0 0 5}$ \\
$\mathrm{N}=$ & 803 & 1204 & 1200 & 2018 & 1092 & 1209 & 1000 & 1203 \\
\hline Gesamt & 23 & 31 & 31 & 32 & 35 & 34 & 34 & 37 \\
\hline Mädchen & 11 & 16 & 19 & 18 & 22 & 21 & 19 & 20 \\
Jungen & 34 & 44 & 44 & 46 & 48 & 47 & 49 & 52 \\
\hline 12-13 J. & 27 & 38 & 37 & 38 & 40 & 37 & 40 & 41 \\
14-15 J. & 21 & 35 & 34 & 38 & 39 & 36 & 34 & 41 \\
$16-17$ J. & 26 & 29 & 32 & 33 & 34 & 34 & 33 & 40 \\
$18-19$ J. & 17 & 21 & 23 & 20 & 28 & 30 & 30 & 25 \\
\hline Hauptschule & 32 & 38 & 36 & 40 & 47 & 46 & 50 & 45 \\
Realschule & 25 & 34 & 34 & 37 & 39 & 39 & 35 & 42 \\
Gymnasium & 16 & 24 & 26 & 24 & 26 & 26 & 26 & 27 \\
\hline
\end{tabular}


Tabelle 8: Besitz eines Handys bei Jugendlichen (JIM-Studie): Prozent aller Jugendlichen, die ein Handy besitzen

\begin{tabular}{rrrrrrrrr}
\hline Jahr & $\mathbf{1 9 9 8}$ & $\mathbf{1 9 9 9}$ & $\mathbf{2 0 0 0}$ & $\mathbf{2 0 0 1}$ & $\mathbf{2 0 0 2}$ & $\mathbf{2 0 0 3}$ & $\mathbf{2 0 0 4}$ & $\mathbf{2 0 0 5}$ \\
$\mathrm{n}=$ & 803 & 1204 & 1200 & 2018 & 1092 & 1209 & 1000 & 1203 \\
\hline Gesamt & 8 & 14 & 49 & 74 & 82 & 87 & 90 & 92 \\
\hline Mädchen & 8 & 14 & 51 & 80 & 87 & 89 & 91 & 94 \\
Jungen & 7 & 14 & 46 & 69 & 77 & 84 & 88 & 90 \\
\hline
\end{tabular}

Neben PC und Konsole werden auch unterschiedliche tragbare Geräte zum Spielen genutzt. In der JIM-Studie liegen hierzu nur Ergebnisse im Hinblick auf die Nutzung des Handys als Spielmedium vor. Der Handybesitz ist in den acht Untersuchungsjahren sowohl bei den Mädchen als auch bei den Jungen von unter $10 \%$ auf über $90 \%$ gestiegen (Tabelle 8). Hinsichtlich der Nutzung von Handyspielen lassen sich solche Tendenzen nicht aufzeigen, denn es liegen nur Daten aus dem Jahr 2003 vor. Diese zeigen, dass ein Großteil der jugendlichen Handybesitzer das Gerät auch zum Spielen nutzt. Bei den Mädchen waren es 79 $\%$, bei den Jungen $77 \%$. Mit zunehmendem Alter werden die Handys jedoch seltener als Spielgerät eingesetzt. Von den jüngsten Handybesitzern (12-13 Jahre) verwenden es $88 \%$ zum Spielen. Bei den älteren Jugendlichen (18-19 Jahre) beträgt der Anteil der Spieler nur noch $66 \%$. Haupt- und Realschüler nutzen Handyspiele etwas häufiger (80 bzw. $82 \%$ ) als Gymnasiasten (73\%).

\section{Entwicklung der Computerspielnutzung bei Kindern}

Anders als bei der JIM-Studie liegen bei der KIM-Studie nur Befragungsdaten von fünf Messzeitpunkten vor. Aus den zuvor bereits genannten Gründen ist es hier ebenfalls notwendig, zunächst die Entwicklung der Computernutzung (Tabelle 9) zu betrachten: Wie schon bei den Jugendlichen nimmt bei den Kindern die Zahl der Computernutzer gleichfalls zu. Auch hier vollzieht sich das Wachstum in allen soziodemografischen Gruppen, allerdings - aufgrund des geringeren Alters der Befragten - auf einem niedrigeren Niveau als bei den Jugendlichen. Aber nicht nur im Vergleich zu den Jugendlichen, auch innerhalb der Kinderstichprobe findet man deutliche Unterschiede zwischen den Jahrgängen. Zwar hat selbst bei den 6- bis 7-jährigen Kindern mittlerweile schon die Hälfte Erfahrungen mit dem Computer gesammelt, bei den ältesten Kindern (12-13 Jahre) ist der Anteil aber bereits auf über $90 \%$ angestiegen. Die zwischen den Geschlechtern im Jahr 1999 noch deutlich vorhandenen Nutzungsklüfte sind im Jahr 2005 weitgehend verschwunden. Die Nutzungsunterschiede zwischen den Schülern der verschiedenen Schulsysteme haben sich im Laufe der Jahre ebenfalls abgeschwächt, sind jedoch im Jahr 2005 noch immer vorhanden. 
Tabelle 9: Computernutzung durch Kinder (KIM-Studie): Prozent aller Kinder, die den Computer zumindest, selten' nutzen (Computer-Kids)

\begin{tabular}{rrrrrr}
\hline Jahr & $\mathbf{1 9 9 9}$ & $\mathbf{2 0 0 0}$ & $\mathbf{2 0 0 2}$ & $\mathbf{2 0 0 3}$ & $\mathbf{2 0 0 5}$ \\
$\mathrm{n}=$ & 1058 & 1228 & 1241 & 1201 & 1203 \\
\hline Gesamt & 51 & 60 & 63 & 70 & 76 \\
\hline Mädchen & 45 & 55 & 59 & 69 & 74 \\
Jungen & 57 & 66 & 67 & 72 & 79 \\
\hline 6-7 J. & 39 & 34 & 39 & 46 & 52 \\
8-9 J. & 45 & 52 & 55 & 63 & 71 \\
10-11 J. & 56 & 70 & 74 & 81 & 84 \\
12-13 J. & 61 & 80 & 82 & 84 & 91 \\
\hline Grundschule & 43 & 47 & 51 & 58 & 64 \\
Hauptschule & 45 & 63 & 62 & 77 & 79 \\
Realschule & 65 & 81 & 80 & 82 & 92 \\
Gymnasium & 79 & 90 & 94 & 92 & 94 \\
\hline
\end{tabular}

Im Unterschied zur JIM-Studie findet man bei der KIM-Studie keine Angaben dazu, wie groß der Gesamtanteil der Kinder ist, die sich regelmäßig mit Computerspielen beschäftigen. Bei den Kindern liegen diese Daten nur jeweils separat für das ,gemeinsame Spiel' und für das ,Einzelspiel' vor. Angegeben sind auch nicht die Zahlen zum Anteil der Spieler, die ,täglich' bzw. ,mehrmals die Woche' am Rechner spielen, wie bei der JIM-Studie, sondern hier wird die Prozentzahl der Kinder ausgewiesen, die ,mindestens einmal die Woche' spielen. Die Antworten lassen sich zudem nicht direkt mit der Auswahlfrage vergleichen, die den Jugendlichen in der JIM-Studie vorgelegt wurde, denn die Kinder wurden nicht aufgefordert zu entscheiden, ob sie häufiger alleine oder häufiger zusammen spielen, sondern die Nutzungshäufigkeit wurde jeweils separat erhoben.

Auch wenn es aufgrund dieser Unterschiede schwer ist, die Ergebnisse direkt miteinander zu vergleichen, lässt sich doch konstatieren, dass die Daten der KIM-Studie ein Bild zeigen, das in mehrfacher Hinsicht von den Ergebnissen der JIM-Studie abweicht (Tabelle 10): Auffällig ist erstens, dass bei den Kindern die Zahl der Spieler nicht nur konstant bleibt, sondern im Untersuchungszeitraum sogar gewachsen ist. Dieses Wachstum ergibt sich aus zwei Tendenzen: Zum einen ist der Anteil der Spieler unter den Computer-Kids von 1999 bis 2005 leicht gestiegen. Einen bedeutenderen Effekt hat aber, dass die Gesamtzahl der computernutzenden Kinder deutlich größer geworden ist.

Der zweite Unterschied bezieht sich auf die beiden Nutzungsmodi. Während bei den Jugendlichen die Bedeutung des Gruppenspiels rückläufig ist, findet man bei den Kindern nicht nur bei den Singleplayern, sondern auch bei den Gruppenplayern einen Anstieg der Nutzerzahlen.

Der dritte Unterschied - dass hier bei beiden Spielvarianten die Jungen vorne liegen ist vermutlich darauf zurückzuführen, dass die Kinder keine Auswahlentscheidungen treffen mussten, sondern jede der beiden Nutzungsformen einzeln abgefragt wurde. Vermutlich 
deswegen ähneln die Ergebnisse in dieser Hinsicht eher den Befunden zur allgemeinen Computerspielnutzung der Jugendlichen. Allerdings ist der Abstand zwischen Jungen und Mädchen nicht annähernd so groß wie bei der JIM-Studie. Das kann teilweise darauf zurückzuführen sein, dass bei der Kinderbefragung nicht nur solche Kinder als Spieler gezählt wurden, die besonders hohe Nutzungsfrequenzen aufweisen, sondern auch diejenigen, die hinsichtlich der Nutzungshäufigkeit eher als ,Durchschnittsspieler' bezeichnet werden können. In dieser Gruppe ist der Anteil der Mädchen meistens höher; Vielspieler sind hingegen häufiger die männlichen Kids.

Tabelle 10: Computerspiel allein oder zusammen (KIM-Studie): Prozent aller ComputerKids, die ,mindestens einmal pro Woche' allein oder mit anderen spielen

\begin{tabular}{|c|c|c|c|c|c|c|}
\hline & Jahr & 1999 & 2000 & 2002 & 2003 & 2005 \\
\hline & $\mathrm{n}=$ & 537 & 740 & 782 & 842 & 919 \\
\hline \multirow{11}{*}{$\begin{array}{l}\text { Nutzung } \\
\text { Computerspiele } \\
\text { allein }\end{array}$} & Gesamt & 58 & 63 & 70 & 70 & 63 \\
\hline & Mädchen & 50 & 58 & 65 & 60 & 55 \\
\hline & Jungen & 63 & 66 & 74 & 79 & 70 \\
\hline & 6-7 J. & 42 & 56 & 64 & 51 & 52 \\
\hline & 8-9 J. & 52 & 65 & 66 & 70 & 58 \\
\hline & $10-11 \mathrm{~J}$. & 61 & 62 & 71 & 69 & 66 \\
\hline & $12-13 \mathrm{~J}$. & 68 & 64 & 73 & 78 & 69 \\
\hline & Grundschule & 49 & 62 & 65 & 65 & 58 \\
\hline & Hauptschule & 58 & 56 & 69 & 71 & 68 \\
\hline & Realschule & 62 & 68 & 81 & 74 & 70 \\
\hline & Gymnasium & 74 & 65 & 69 & 72 & 61 \\
\hline \multirow{11}{*}{$\begin{array}{l}\text { Nutzung } \\
\text { Computerspiele } \\
\text { mit anderen } \\
\text { gemeinsam }\end{array}$} & Gesamt & 48 & 46 & 51 & 53 & 50 \\
\hline & Mädchen & 40 & 42 & 43 & 47 & 45 \\
\hline & Jungen & 54 & 49 & 59 & 59 & 56 \\
\hline & 6-7 J. & 40 & 40 & 44 & 40 & 43 \\
\hline & 8-9 J. & 37 & 42 & 51 & 49 & 45 \\
\hline & $10-11 \mathrm{~J}$. & 59 & 46 & 50 & 56 & 54 \\
\hline & $12-13 \mathrm{~J}$. & 52 & 51 & 56 & 59 & 54 \\
\hline & Grundschule & 39 & 44 & 48 & 47 & 45 \\
\hline & Hauptschule & 60 & 45 & 58 & 67 & 58 \\
\hline & Realschule & 50 & 54 & 53 & 51 & 53 \\
\hline & Gymnasium & 62 & 44 & 48 & 58 & 50 \\
\hline
\end{tabular}


Ein anderer Indikator für das Spielverhalten ist die Nutzungsdauer. Schaut man auf die Dauer, die mit dem Computerspiel verbracht wird, dann stellt man fest, dass unter den Jungen mehr Intensivspieler zu finden sind als unter den Mädchen. Wenn der Computer einmal eingeschaltet ist, dann neigen vor allem die Jungen zu langen Nutzungszeiten (Tabelle 11). Der Anteil der Jungen, die sich in einer normalen Spielsession länger als eine Stunde mit Computerspielen beschäftigen, ist ungefähr doppelt so hoch wie der Prozentanteil bei den Mädchen. Diese Relation hat sich insgesamt nur geringfügig verändert. Eine Ausnahme bilden dabei nur die ganz jungen Kids. Bei ihnen ist der Anteil der Intensivspieler im Beobachtungszeitraum kontinuierlich gestiegen.

Tabelle 11: Spieldauer von Computerspielen an Nutzungstagen (KIM-Studie): Prozent aller Computer-Kids, die an Nutzungstagen, länger als 60 Minuten’ spielen

\begin{tabular}{rrrrr}
\hline Jahr & $\mathbf{2 0 0 0}$ & $\mathbf{2 0 0 2}$ & $\mathbf{2 0 0 3}$ & $\mathbf{2 0 0 5}$ \\
$\mathrm{n}=$ & 740 & 782 & 842 & 919 \\
\hline Gesamt & 15 & 17 & 19 & 18 \\
\hline Mädchen & 10 & 11 & 13 & 12 \\
Jungen & 20 & 21 & 24 & 23 \\
\hline 6-7 J. & 3 & 5 & 11 & 11 \\
8-9 J. & 11 & 11 & 19 & 11 \\
$10-11 \mathrm{~J}$. & 18 & 15 & 28 & 18 \\
$12-13 \mathrm{~J}$. & 20 & 27 & & 25 \\
\hline
\end{tabular}

$\mathrm{Zu}$ den Genrepräferenzen der Kinder liegen erst drei Messzeitpunkte vor. Anders als bei den Jugendlichen findet man bei den Kindern keine klaren Favoriten (Tabelle 12). Fast alle abgefragten Genres wurden in den drei Befragungen jeweils von rund 15 bis $30 \%$ der Kinder als Lieblingsgenre genannt. Jedoch gibt es auch Gemeinsamkeiten: Sowohl Action- als auch Simulationsspiele werden bereits im Kindesalter von den Jungen häufiger als Lieblingsgenre genannt. Hinsichtlich der Sportspiele zeigt sich, dass sie eindeutig von den männlichen Kids präferiert werden. Ob diese Vorliebe auch bei den männlichen Jugendlichen festzustellen ist, lässt sich nicht abschließend beantworten, da Vergleichsdaten aus der JIM-Studie fehlen. Ergebnisse von Klink et al. (in diesem Band) sprechen allerdings dafür. Die Genrepräferenzen der Mädchen zwischen sechs und 13 Jahren liegen eindeutig bei Lernspielen, Fun-Games und Jump'n'Runs. Das starke Interesse der Mädchen an Strategiespielen zeigt sich bereits im kindlichen Alter. Allerdings unterscheiden sie sich in dieser Hinsicht nicht von den Jungen. Der Vorsprung gegenüber den männlichen Spielern entwickelt sich erst in der Jugend.

Klare Trends, die darauf hindeuten, dass sich die Genrepräferenzen der ComputerKids verändern, lassen sich den drei Messzeitpunkten nicht entnehmen. 
Tabelle 12: Computerspielgenrepräferenzen der Kinder (KIM-Studie): Prozent der Computer-Kids, die das jeweilige Genre genannt haben

\begin{tabular}{|c|c|c|c|c|}
\hline Lieblingsspiel & Jahr & 2002 & 2003 & 2005 \\
\hline bis zu 3 Nennungen & $\mathrm{n}=$ & 764 & 807 & 846 \\
\hline \multirow[t]{2}{*}{ Strategie } & Mädchen & 30 & 32 & 33 \\
\hline & Jungen & 34 & 35 & 36 \\
\hline \multirow[t]{2}{*}{ Action } & Mädchen & 22 & 28 & 17 \\
\hline & Jungen & 24 & 33 & 25 \\
\hline \multirow[t]{2}{*}{ Simulation } & Mädchen & 21 & 27 & 29 \\
\hline & Jungen & 31 & 46 & 42 \\
\hline \multirow[t]{2}{*}{ Adventure } & Mädchen & 19 & 14 & 11 \\
\hline & Jungen & 19 & 19 & 13 \\
\hline \multirow[t]{2}{*}{ Sportspiel } & Mädchen & 7 & 9 & 4 \\
\hline & Jungen & 20 & 25 & 23 \\
\hline \multirow[t]{2}{*}{ Jump'n'Run } & Mädchen & 22 & 17 & 27 \\
\hline & Jungen & 17 & 13 & 19 \\
\hline \multirow[t]{2}{*}{ Fun / Gesellschaftsspiele } & Mädchen & 27 & 31 & 28 \\
\hline & Jungen & 18 & 24 & 21 \\
\hline \multirow[t]{2}{*}{ Lernspiele } & Mädchen & 19 & 15 & 20 \\
\hline & Jungen & 10 & 8 & 10 \\
\hline
\end{tabular}

Um die Entwicklung der Onlinespielnutzung der Kinder angemessen zu interpretieren, muss wiederum zunächst die generelle Onlinenutzung betrachtet werden. Auch hier ist der Befund eindeutig: Wie schon bei den Jugendlichen hat sich auch bei den Kindern die Onlinenutzung erheblich ausgeweitet (Tabelle 13). Der Anteil der Computer-Kids, die zumindest , selten' online gehen, hat sich von $31 \%$ auf $66 \%$ in fünf Jahren mehr als verdoppelt. Diese Wachstumsraten findet man in allen soziodemographischen Gruppen. Nutzungsklüfte, die auf das Geschlecht zurückgeführt werden können, sind nicht erkennbar. Die Unterschiede zwischen den Schülern der verschiedenen Schulsysteme sind in den Jahren geringer geworden und mittlerweile so gut wie verschwunden. Einzig die altersbedingten Unterschiede bestehen weiter: Von den 6- bis 7-jährigen Computer-Kids haben 2005 gut $2 / 3$ noch keine Onlineerfahrungen, bei den 12- bis 13-jährigen ist dies nur noch eine Minderheit von unter $20 \%$. 
Tabelle 13: Onlinenutzung durch Kinder (KIM-Studie): Prozent aller Computer-Kids, die zumindest ,selten' online gehen (Online-Kids)

\begin{tabular}{rrrrr}
\hline Jahr & $\mathbf{2 0 0 0}$ & $\mathbf{2 0 0 2}$ & $\mathbf{2 0 0 3}$ & $\mathbf{2 0 0 5}$ \\
$\mathrm{n}=$ & 740 & 782 & 842 & 919 \\
\hline Gesamt & 31 & 52 & 60 & 66 \\
\hline Mädchen & 30 & 51 & 62 & 66 \\
Jungen & 32 & 53 & 58 & 66 \\
\hline 6-7 J. & 16 & 36 & 38 & 32 \\
8-9 J. & 24 & 45 & 52 & 51 \\
$10-11 \mathrm{~J}$. & 33 & 49 & 63 & 71 \\
$12-13 \mathrm{~J}$. & 37 & 66 & 71 & 83 \\
\hline Grundschule & 24 & 41 & 48 & 46 \\
Hauptschule & 32 & 53 & 60 & 79 \\
Realschule & 28 & 62 & 70 & 80 \\
Gymnasium & 49 & & 80 & 86 \\
\hline
\end{tabular}

Wie schon bei den Jugendlichen hat sich auch bei den Kindern der Anteil der Onlinespieler im Untersuchungszeitraum nicht wesentlich verändert (Tabelle 14). Und auch hier findet man in den verschiedenen soziodemographischen Gruppen zum Teil erhebliche Schwankungen zwischen den Jahren. Dies unterstützt die Vermutung, dass bei Kindern und Jugendlichen begriffliche Unklarheiten vorhanden sind, was genau mit einem Onlinegame gemeint ist. Hinzu kommt, dass sich gerade bei den Onlinespielen die Angebotssituation rasch verändert hat, was die Variationen sicherlich mit erklären kann.

Auf den ersten Blick scheinen die Daten somit auf zwei widersprüchliche Eigenschaften dieser Nutzergruppe hinzudeuten: Zum einen handelt es sich bei den Kindern offenbar um eine eher volatile Nutzerschaft, zum anderen erweist sich die Gruppe im Aggregat als weitgehend stabil. Dabei sollte man jedoch nicht vergessen, dass sich - aufgrund der größer werdenden Zahl von Computer- und Online-Kids - hinter der prozentualen Stagnation eine wachsende Anzahl von Kindern verbirgt, die sich regelmäßig mit Onlinespielen beschäftigt.

Auch bei den Kindern wurde neben der Nutzung des PCs als Spielmedium gleichfalls erfragt, ob sie die Möglichkeit haben, mit einer Konsole zu spielen. Daten zum Handyspiel oder zur Nutzung anderer mobiler Spielgeräte wurden allerdings nicht erhoben. Nicht nur unter Jugendlichen, sondern auch bei Kindern haben die Spielkonsolen im Jahr 2005 eine größere Verbreitung als im Jahr 1999. Die unterschiedliche Ausstattung von Jungen und Mädchen mit Konsolen, die bei den Jugendlichen festzustellen war, ist ebenfalls bei den Kindern zu beobachten (Tabelle 15). Die Prozentwerte zur Verbreitung von Konsolen unter den Schülern verschiedener Schultypen variieren zwischen den Erhebungen relativ stark. Hier lassen sich keine eindeutigen Trends ablesen. Klar erkennbar ist aber, dass bei Kindern unter zehn Jahren der Anteil der Konsolenbesitzer in allen Erhebungen geringer ist. Betrachtet man die Ergebnisse der JIM- und KIM-Studien im Zusammenhang, dann ist ersichtlich, dass Konsolen das Computer-Spielzeug der 10- bis 17-Jährigen sind. 
Tabelle 14: Nutzung von Onlinespielen durch Kinder (KIM-Studie): Prozent der OnlineKids, die ,mindestens einmal pro Woche' Onlinespiele nutzen

\begin{tabular}{rrrrr}
\hline Jahr & $\mathbf{2 0 0 0}$ & $\mathbf{2 0 0 2}$ & $\mathbf{2 0 0 3}$ & $\mathbf{2 0 0 5}$ \\
$\mathrm{n}=$ & 227 & 406 & 505 & $624^{6}$ \\
\hline Gesamt & 22 & 15 & 18 & 23 \\
\hline Mädchen & 24 & 11 & 13 & 17 \\
Jungen & 20 & 17 & 23 & 28 \\
\hline 6-7 J. & 20 & 7 & 16 & 23 \\
$8-9$ J. & 22 & 13 & 18 & 14 \\
$10-11 \mathrm{~J}$. & 17 & 12 & 20 & 21 \\
$12-13 \mathrm{~J}$. & 25 & 19 & 17 & 26 \\
\hline Grundschule & 21 & 10 & 20 & 16 \\
Hauptschule & 18 & 10 & 18 & 30 \\
Realschule & 34 & 15 & 15 & 18 \\
Gymnasium & 17 & & & \\
\hline
\end{tabular}

Tabelle 15: Besitz einer Spielkonsole bei Kindern (KIM-Studie): Prozent aller Kinder, die eine Spielkonsole besitzen (nach Angabe der Mutter)

\begin{tabular}{rrrrrr}
\hline Jahr & $\mathbf{1 9 9 9}$ & $\mathbf{2 0 0 0}$ & $\mathbf{2 0 0 2}$ & $\mathbf{2 0 0 3}$ & $\mathbf{2 0 0 5}$ \\
$\mathrm{n}=$ & 1058 & 1228 & 1241 & 1201 & 1203 \\
\hline Gesamt & 28 & 26 & 25 & 27 & 35 \\
\hline Mädchen & 22 & 23 & 18 & 26 & 26 \\
Jungen & 34 & 30 & 32 & 30 & 44 \\
\hline 6-7 J. & 19 & 16 & 24 & 23 & 23 \\
8-9 J. & 25 & 25 & 18 & 24 & 24 \\
10-11 J. & 35 & 28 & 27 & 40 & 40 \\
12-13 J. & 34 & 34 & 32 & 47 & 47 \\
\hline Grundschule & 23 & 21 & 21 & 22 & 27 \\
Hauptschule & 35 & 35 & 21 & 37 & 41 \\
Realschule & 34 & 38 & 38 & 26 & 44 \\
Gymnasium & 48 & 31 & 37 & 31 & 44 \\
\hline
\end{tabular}

6 Veränderte Fragestellung: bis 2003 „Netzspiele spielen“, ab 2005: „,mit anderen Internetnutzern Spiele spielen". 


\section{$4 \quad$ Entwicklung der Computerspielnutzung bei Erwachsenen}

Um die Entwicklung der Computerspielnutzung bei den Erwachsenen zu rekonstruieren, wird auf die ACTA zurückgegriffen. Die Daten der ACTA wurden allerdings anders aufbereitet als die der JIM- und KIM-Studie. Die Prozentzahlen beziehen sich bei der ACTA immer auf die Gesamtbevölkerung (14-64 Jahre). Deswegen ist es bei den Erwachsenen nicht zwingend nötig, sich die Entwicklung der allgemeinen Computer-, Online- oder Handynutzung vorher separat anzusehen.

Anders als bei den Kindern und ähnlich wie bei den Jugendlichen hat sich die Computerspielnutzung der Erwachsenen in der Zeit von 1998 bis 2005 nur geringfügig verändert. Die Tendenz ist zwar nicht linear, insgesamt sprechen die Daten aber für eine gewisse $\mathrm{Zu}$ nahme der Nutzerzahlen. ${ }^{7}$ Dieser Zuwachs ist vor allem auf die Männer sowie auf die jüngeren Personen zurückzuführen. Insbesondere bei den 14- bis 24-Jährigen (die teilweise auch im Rahmen der JIM-Studie befragt wurden) ist ein deutlicher Anstieg zu verzeichnen. Bei den 25- bis 39-Jährigen fällt der Zuwachs schon deutlich schwächer aus und bei den über 40-Jährigen sind die Anteile weitgehend konstant. Die schon 1998 deutlich vorhandenen altersbedingten Nutzungsunterschiede haben sich somit noch weiter verstärkt. Das Gleiche gilt für die Nutzungsdiskrepanz zwischen Männer und Frauen. Auch der Abstand zwischen den Geschlechtern hat sich tendenziell vergrößert.

Die Tatsache, dass insbesondere bei den Jüngeren ein Anstieg der Nutzerzahlen zu verzeichnen ist, korrespondiert mit den deutlichen Zuwachsraten bei den ungebundenen Personen ohne Lebensgefährten sowie bei den Befragten, die sich noch in Ausbildung befinden. 1998 war sowohl bei den Verheirateten als auch bei den Ungebundenen (mit oder ohne Lebensgefährten) der Nutzeranteil gleich groß. 2005 sind nun erhebliche Unterschiede festzustellen.

Durchaus bemerkenswert ist sicherlich auch, dass die bei den Kindern und Jugendlichen ermittelten Nutzungsunterschiede zwischen den Bildungsgruppen (bzw. Schultypen) sich bei den Erwachsenen ganz anders darstellen. Bei den Erwachsenen ist der Anteil der Computerspieler in der Gruppe der höher Gebildeten größer. Dieser Effekt ist zwar im Wesentlichen darauf zurückzuführen, dass Bildung und Alter miteinander korrelieren, dennoch bleibt festzuhalten, dass die erwachsenen Spieler keinesfalls überwiegend zu den geringer Gebildeten gehören.

Während sich die Nutzungsunterschiede zwischen den Geschlechtern und den Altersgruppen im Untersuchungszeitraum vergrößert haben, findet man zwischen den Einkommensgruppen einen gegenläufigen Trend: 1998 war der Anteil der Spieler bei Personen mit hohem Einkommen deutlich höher als bei denen mit niedrigem Einkommen; im Jahr 2005 sind keine einkommensbedingten Unterschiede mehr festzustellen. Die Spiele sind breitflächig in die Gesellschaft diffundiert. Finanzielle Restriktionen sind demnach kein Hinderungsgrund mehr, sich mit Computerspielen zu beschäftigen.

Wortlaut des Items: „Wozu nutzen Sie persönlich den/die Computer zu Hause? Was von dem, was hier steht, machen Sie mit dem Computer?“ Antwortvorgabe: ,Spielen’. 
Tabelle 16: Computerspielnutzung durch Erwachsene (ACTA): Prozent der Befragten, die ,Spielen’ als Tätigkeit genannt haben, zu der sie den Computer nutzen

\begin{tabular}{|c|c|c|c|c|c|c|c|c|}
\hline Jahr & 1998 & 1999 & 2000 & 2001 & 2002 & 2003 & 2004 & 2005 \\
\hline $\mathrm{n}=$ & 9558 & 10132 & 10012 & 10039 & 10507 & 10424 & 10287 & 10329 \\
\hline Gesamt & 32 & 35 & 28 & 31 & 32 & 35 & 34 & 37 \\
\hline Frauen & 29 & 32 & 22 & 27 & 27 & 29 & 30 & 31 \\
\hline Männer & 35 & 37 & 34 & 36 & 38 & 40 & 39 & 42 \\
\hline 14-17 Jahre & 55 & 60 & 58 & 67 & 65 & 76 & 71 & 74 \\
\hline 18-24 Jahre & 40 & 48 & 44 & 50 & 52 & 56 & 56 & 57 \\
\hline 25-39 Jahre & 34 & 38 & 34 & 38 & 38 & 39 & 41 & 42 \\
\hline 40-49 Jahre & 38 & 39 & 26 & 29 & 29 & 30 & 31 & 33 \\
\hline 50-64 Jahre & 16 & 17 & 11 & 12 & 17 & 17 & 15 & 17 \\
\hline verheiratet & 32 & 34 & 23 & 25 & 28 & 27 & 27 & 30 \\
\hline mit Lebensgefährten & 31 & 34 & 31 & 35 & 33 & 36 & 38 & 37 \\
\hline ohne Lebensgefährten & 32 & 36 & 36 & 40 & 40 & 45 & 43 & 46 \\
\hline Hauptschule & 23 & 31 & 17 & 20 & 23 & 25 & 27 & 28 \\
\hline Realschule & 36 & 52 & 34 & 38 & 39 & 40 & 39 & 41 \\
\hline Abitur & 44 & 44 & 39 & 43 & 41 & 43 & 40 & 44 \\
\hline in Ausbildung & 54 & 58 & 55 & 63 & 60 & 66 & 65 & 69 \\
\hline berufstätig & 32 & 36 & 28 & 31 & 33 & 35 & 36 & 36 \\
\hline nicht berufstätig & 20 & 22 & 14 & 16 & 21 & 23 & 21 & 24 \\
\hline unter 1000 Euro $^{8}$ & 16 & 21 & 19 & 22 & 27 & 29 & 32 & 33 \\
\hline 1000-2000 Euro & 25 & 27 & 25 & 27 & 28 & 32 & 31 & 34 \\
\hline 2000-3500 Euro & 39 & 42 & 31 & 34 & 36 & 37 & 37 & 39 \\
\hline über 3500 Euro & 46 & 47 & 35 & 39 & 36 & 37 & 36 & 37 \\
\hline
\end{tabular}

Im Vergleich zur allgemeinen Computerspielnutzung ist die Nutzung von Onlinespielen ${ }^{9}$ sehr deutlich und kontinuierlich angestiegen, von $2 \%$ im Jahr 1998 auf 17 \% im Jahr 2005 (Tabelle 17). Das Wachstum ist in allen hier unterschiedenen Gruppen zu verzeichnen, vollzieht sich aber mit unterschiedlicher Dynamik: Besonders große Zuwachsraten sind bei den Männern, den höher Gebildeten und vor allem bei den jüngeren Befragten zu verzeichnen.

Einkommenskategorien bis einschließlich 2001 in DM: unter 2000, 2000-3999, 4000-5999, 6000 und mehr. Wortlaut des Items: „Es gibt ja ganz verschiedene Angebote im Internet. Hier auf diesen Karten steht eine Auswahl. Bitte verteilen Sie die Karten entsprechend auf das Bildblatt hier." Onlinespieler sind diejenigen Befragten, die ,häufig/regelmäßig (...)', , ab und zu (...)’ oder ,nur ganz selten Spiele gegen Computer oder andere Nutzer' im Internet machen. 
Tabelle 17: Nutzung von Onlinespielen durch Erwachsene (ACTA): Prozent der Befragten, die mindestens , selten' Onlinespiele nutzen

\begin{tabular}{|c|c|c|c|c|c|c|c|c|}
\hline Jahr & 1998 & 1999 & 2000 & 2001 & 2002 & 2003 & 2004 & 2005 \\
\hline $\mathrm{n}=$ & 9558 & 10132 & 10012 & 10039 & 10507 & 10424 & 10287 & 10329 \\
\hline Gesamt & 2 & 2 & 4 & 12 & 15 & 16 & 16 & 17 \\
\hline Frauen & 1 & 1 & 2 & 8 & 10 & 11 & 11 & 12 \\
\hline Männer & 3 & 3 & 6 & 16 & 19 & 21 & 20 & 23 \\
\hline 14-17 Jahre & 4 & 5 & 12 & 34 & 39 & 44 & 43 & 47 \\
\hline 18-24 Jahre & 3 & 5 & 9 & 26 & 30 & 34 & 34 & 36 \\
\hline 25-39 Jahre & 2 & 2 & 5 & 16 & 16 & 17 & 17 & 19 \\
\hline 40-49 Jahre & 1 & 1 & 2 & 8 & 11 & 12 & 10 & 11 \\
\hline 50-64 Jahre & 0 & 0 & 1 & 3 & 5 & 6 & 5 & 6 \\
\hline verheiratet & 1 & 1 & 2 & 8 & 10 & 10 & 9 & 10 \\
\hline mit Lebensgefährten & 2 & 3 & 5 & 15 & 15 & 16 & 16 & 20 \\
\hline ohne Lebensgefährten & 3 & 3 & 7 & 20 & 22 & 25 & 24 & 26 \\
\hline Hauptschule & 1 & 1 & 2 & 8 & 10 & 12 & 11 & 13 \\
\hline Realschule & 2 & 4 & 4 & 15 & 18 & 17 & 18 & 19 \\
\hline Abitur & 2 & 2 & 7 & 18 & 19 & 21 & 19 & 22 \\
\hline in Ausbildung & 5 & 5 & 13 & 34 & 35 & 39 & 40 & 43 \\
\hline berufstätig & 2 & 1 & 3 & 12 & 15 & 16 & 15 & 17 \\
\hline nicht berufstätig & 1 & 0 & 1 & 4 & 7 & 8 & 8 & 9 \\
\hline unter 1000 Euro & 1 & 2 & 3 & 10 & 11 & 15 & 15 & 16 \\
\hline 1000-2000 Euro & 2 & 1 & 4 & 11 & 12 & 15 & 14 & 15 \\
\hline 2000-3500 Euro & 2 & 2 & 4 & 13 & 16 & 16 & 16 & 19 \\
\hline über 3500 Euro & 2 & 3 & 5 & 15 & 19 & 18 & 18 & 18 \\
\hline
\end{tabular}

Das führt dazu - wie schon bei den Computerspielen allgemein -, dass sich auch der Nutzeranteil bei den Ungebundenen sowie bei denen, die sich in der Ausbildung befinden, dementsprechend schnell entwickelt hat. In Folge dessen sind die Diskrepanzen, die mit diesen Personenmerkmalen verbunden sind, im Jahr 2005 erheblich größer. Bemerkenswert - gerade im Vergleich zur Entwicklung der allgemeinen Computerspielnutzung - ist sicherlich, dass sich die Ausweitung der Onlinespielnutzung in allen Einkommensklassen in nahezu gleicher Geschwindigkeit vollzogen hat (vgl. Quandt \& Wimmer in diesem Band).

Was für die Onlinespiele gesagt wurde, lässt sich nahezu eins zu eins auf die Handyspiele übertragen: In allen Bevölkerungsgruppen ist eine schnelle Verbreitung dieser Spiele 
zu verzeichnen. ${ }^{10}$ Bei den Handyspielen sind es ebenfalls die Jüngeren, bei denen die Handyspiele schnell an Popularität gewonnen haben (Tabelle 18). Unterschiede zur Diffusion der Onlinespiele findet man hinsichtlich des Geschlechts. Die Handyspiele sind der einzige Bereich der Spielwelt, bei dem sich kaum Nutzungsunterschiede zwischen den Geschlechtern zeigen. Dies gilt nicht nur für Mädchen und Jungen (JIM-Studie), sondern auch für Frauen und Männer. Anders als bei den Onlinespielen findet man bei den Handyspielen keine Unterschiede zwischen den Bildungsgruppen, und auch das Einkommen ist für die Nutzung von Handyspielen irrelevant.

Tabelle 18: Nutzung von Handyspielen durch Erwachsene (ACTA): Prozent der Befragten, die ,Spiele' als Funktion des Handys nannten, die sie ,tatsächlich nutzen'

\begin{tabular}{rrrrrrr}
\hline Jahr & $\mathbf{2 0 0 0}$ & $\mathbf{2 0 0 1}$ & $\mathbf{2 0 0 2}$ & $\mathbf{2 0 0 3}$ & $\mathbf{2 0 0 4}$ & $\mathbf{2 0 0 5}$ \\
$\mathrm{n}=$ & 10012 & 10039 & 10507 & 10424 & 10287 & 10329 \\
\hline Gesamt & 8 & 16 & 21 & 25 & 25 & 22 \\
\hline Frauen & 6 & 15 & 19 & 24 & 23 & 20 \\
Männer & 11 & 18 & 23 & 27 & 26 & 24 \\
\hline 14-17 Jahre & 17 & 43 & 55 & 63 & 67 & 63 \\
18-24 Jahre & 22 & 38 & 47 & 53 & 52 & 48 \\
25-39 Jahre & 10 & 19 & 25 & 30 & 28 & 26 \\
40-49 Jahre & 5 & 11 & 13 & 18 & 17 & 14 \\
50-64 Jahre & 2 & 4 & 6 & 7 & 8 & 6 \\
\hline verheiratet & 5 & 10 & 13 & 16 & 15 & 13 \\
mit Lebensgefährten & 11 & 21 & 24 & 31 & 29 & 25 \\
ohne Lebensgefährten & 13 & 26 & 32 & 38 & 37 & 34 \\
\hline Hauptschule & 6 & 13 & 18 & 21 & 21 & 20 \\
Realschule & 10 & 19 & 24 & 29 & 28 & 24 \\
Abitur & 9 & 18 & 22 & 27 & 26 & 23 \\
\hline in Ausbildung & 19 & 40 & 50 & 57 & 59 & 53 \\
berufstätig & 8 & 16 & 22 & 26 & 25 & 21 \\
nicht berufstätig & 4 & 8 & 11 & 15 & 13 & 14 \\
\hline unter 1000 Euro & 7 & 15 & 21 & 28 & 27 & 24 \\
1000-2000 Euro & 8 & 16 & 20 & 24 & 23 & 22 \\
2000-3500 Euro & 9 & 16 & 22 & 26 & 26 & 23 \\
\hline 3500 Euro & 10 & 18 & 21 & 26 & 24 & 20 \\
\hline & & & & 26 & 26 \\
\hline
\end{tabular}

10 Fragestellung: „Viele Handys haben ja eine Reihe von Zusatzfunktionen oder besonderen Ausstattungen. Hier auf dieser Liste haben wir mal aufgeschrieben, was es gibt. Was davon nutzen Sie? Ich meine jetzt alles, was Sie tatsächlich nutzen.“ Handyspieler sind jene, die ,Spielefunktion, eingebaute Spiele’ antworten. 


\section{Resümee}

Durch die Gegenüberstellung der Daten aus den drei Studien war es möglich, ein facettenreiches Bild der Computerspielnutzung von Kindern, Jugendlichen und Erwachsenen zu entwerfen. Es wird darauf verzichtet, einzelne Befunde hier noch einmal herauszugreifen und hervorzuheben. Wichtiger erscheint es, die grundlegenden Vorzüge und Grenzen der vorhandenen Daten noch einmal deutlich zu machen.

Vor allem die Möglichkeit, durch die Längsschnittperspektive Entwicklungstrends aufzeigen zu können, hat sich als sehr nützlich erwiesen: Bei einigen Angeboten und einigen Nutzersegmenten ist nämlich die Entwicklung überraschend undramatisch verlaufen (z.B. hinsichtlich des generellen Anteils der jugendlichen Computerspielernutzer), was die Aufregung um die immer wieder hervorgehobene rasante Entwicklung des Marktes etwas relativiert. In anderen Bereichen wiederum (z.B. bei der Anzahl der Onlinespieler) findet man jedoch genau diese stürmische Ausweitung der Nutzerzahlen. Wieder andere Zeitreihen verdeutlichen, dass keinesfalls nur lineare Trends zu beobachten sind, sondern teilweise erhebliche Schwankungen von Jahr zu Jahr auftreten. Ein Beispiel hierfür sind die Genrepräferenzen der Jugendlichen, aber auch die Ergebnisse zur allgemeinen Computerspielnutzung der Erwachsenen. Diese Schwankungen und die zum Teil gegenläufigen Trends bei unterschiedlichen Angebotsformen und in verschiedenen Nutzergruppen zeigen, wie behutsam einzelne Querschnittsstudien interpretiert werden sollten. Vor allem verbietet es sich, aus Unterschieden in Querschnittanalysen auf Trends zu schließen.

Andererseits ist aber auch zu konstatieren, dass die vorliegenden Daten zwar einen breiten Überblick über die Nutzung von Computerspielen in Deutschland vermitteln, dass auf diesem Weg jedoch kaum etwas über Beweggründe, Spielweisen und Umstände der Nutzung herausgefunden werden kann. Dafür bedarf es ergänzender Studien, die einzelne Spielergruppen genauer betrachten oder spezifische Genres und Spielformen detailliert analysieren.

\section{Literaturverzeichnis}

Allensbacher Computer- und Technik-Analyse (o.J.). URL: http://www.acta-online.de [7.1.2006]

Eimeren, B. v. \& Frees, B. (2005): ARD/ZDF-Online-Studie 2005: Nach dem Boom: Größter Zuwachs in internetfernen Gruppen. Media Perspektiven, (8), 362-379.

Emmer, M. (2005): Politische Mobilisierung durch das Internet. München: R. Fischer.

Feierabend, S. \& Klingler, W. (2003a): Fünf Jahre JIM-Studie Jugend, Information, (Multi-) Media: Medienverhalten Jugendlicher in Deutschland. Media Perspektiven, (10), 450-462.

Feierabend, S. \& Klingler, W. (2003b): Kinder und Medien 2002. Ergebnisse der Studie KIM 2002 zum Medienumgang Sechs- bis 13-Jähriger in Deutschland. Media Perspektiven, (6), 278-289.

Feierabend, S. \& Klingler, W. (1999): Kinder und Medien 1999. Ergebnisse der Studie KIM 99 zur Mediennutzung von Kindern. Media Perspektiven, (12), 610-625.

Feierabend, S. \& Klingler, W. (2000): Jugend, Information, (Multi-)Media 2000. Aktuelle Ergebnisse der JIM-Studie zum Medienumgang Zwölf- bis 19-Jähiger. Media Perspektiven, (11), 517-527.

Feierabend, S. \& Klingler, W. (2001): Kinder und Medien 2000: PC/Internet gewinnen an Bedeutung. Ergebnisse der Studie KIM 2000 zur Mediennutzung von Kindern. Media Perspektiven, (7), 345-357.

Feierabend, S. \& Klingler, W. (2002): Medien- und Themeninteressen Jugendlicher. Ergebnisse der JIM-Studie 2001 zum Medienumgang Zwölf- bis 19-Jähriger. Media Perspektiven, (1), 9-21. 
Feierabend, S. \& Rathgeb, T. (2005): Medienverhalten Jugendlicher 2004. Neuste Ergebnisse der JIM-Studie Jugend, Information, (Multi-)Media. Media Perspektiven, (7), 320-332.

Klimmt, C. (2004): Computer- und Videospiele. In: R. Mangold, P. Vorderer \& G. Bente (Hrsg.): Lehrbuch der Medienpsychologie. Göttingen u.a.: Hogrefe, 695-716.

Langness, A., Leven, I. \& Hurrelmann, K. (2006): Jugendliche Lebenswelten: Familie, Schule, Freizeit. In: K. Hurrelmann \& M. Albert (2006): Jugend 2006. 15. Shell Jugendstudie. Eine pragmatische Generation unter Druck. Frankfurt am Main: S. Fischer, 49-102.

Medienpädagogischer Forschungsverband Südwest (o.J.): JIM-Studie / KIM-Studie. URL: http://www.mpfs.de [7.1.2006]

Opaschowski, H. W. (1999): Generation @. Die Medienrevolution entläßt ihre Kinder: Leben im Informationszeitalter. Hamburg: British-American Tobacco.

Schlütz, D. (2002): Bildschirmspiele und ihre Faszination. Zuwendungsmotive, Gratifikationen und Erleben interaktiver Medienangebote. München: R. Fischer.

Theunert, H., Demmler, K. \& Kirchhoff, A. (2002): Vom Ego-Shooter zum Amokläufer? Fragen an PC- und Netzspieler. medien \& erziehung, (46), 138-142. 